\title{
Association of retinal vessel attenuation with visual function in eyes with retinitis pigmentosa
}

This article was published in the following Dove Press journal:

Clinical Ophthalmology

12 August 2014

Number of times this article has been viewed

\author{
Satoko Nakagawa \\ Akio Oishi \\ Ken Ogino \\ Yukiko Makiyama \\ Masafumi Kurimoto \\ Nagahisa Yoshimura \\ Department of Ophthalmology \\ and Visual Sciences, Kyoto University \\ Graduate School of Medicine, \\ Kyoto, Japan
}

Purpose: To investigate the association between visual changes and retinal vessel attenuation in patients with retinitis pigmentosa (RP).

Design: A retrospective, longitudinal, observational cohort study.

Methods: We analyzed 45 eyes from 45 subjects who were followed-up for $\geq 3$ years at our clinic. Using the computer-based Interactive Vessel Analysis program, central retinal artery equivalent (CRAE) and central retinal vein equivalent (CRVE) were determined. Age- and sex-matched controls from normal subjects were selected from our archived fundus photograph library. Visual acuity, visual field area (Goldmann perimetry, V4e white test light), mean deviation (Humphrey perimetry, central 10-2 program), and central macular thickness (optical coherence tomography) were analyzed for correlations with CRAE and CRVE.

Results: Both CRAE and CRVE were significantly decreased in RP eyes $(94.9 \pm 13.5 \mu \mathrm{m}$ and $155.6 \pm 20.0 \mu \mathrm{m}$, respectively) compared with control eyes $(138.1 \pm 14.7 \mu \mathrm{m}$ and $215.0 \pm 20.4 \mu \mathrm{m}$, respectively, both $P<0.001)$. After 3 years of follow-up, visual field area was associated with both CRAE $(r=0.584, P<0.01)$ and CRVE $(r=0.500, P=0.008)$. A significant association was also observed between mean deviation and CRAE $(r=0.298, P=0.047)$. In eyes with RP, a narrower vessel caliber at baseline was associated with a larger decline in visual acuity over the 3-year follow-up interval (CRAE: $r=-0.344, P=0.021$; CRVE: $r=-0.314, P=0.035$ ).

Conclusion: Retinal vessel caliber is associated with some visual functions in patients with RP. Keywords: retinitis pigmentosa, retinal vascular caliber, central retinal artery equivalent, central retinal vein equivalent

\section{Introduction}

Retinitis pigmentosa (RP) is a term used for a group of disorders characterized by an inherited progressive degeneration of photoreceptor cells. ${ }^{1}$ The three hallmark fundus characteristics of RP are scattered pigment in the form of bone spicules, attenuated blood vessels, and waxy pale discs. ${ }^{2}$ Among these, only vessel attenuation can be seen in the early stages of the disease. ${ }^{3}$

Alteration of retinal and choroidal vessels and their circulation has been investigated in patients with RP. Many experimental and clinical studies, using laser Doppler flowmetry, ${ }^{4-8}$ magnetic resonance imaging,,${ }^{9,10}$ and/or ocular pulse amplitude, ${ }^{11}$ showed choroidal and retinal blood flow reductions in patients with RP and/or in animal models of RP. Some of these studies quantified the attenuations in retinal vessels. ${ }^{5,6}$ Another study focused on retinal vessel tortuosity and reported that retinal vessels had a higher tortuosity in eyes with RP than in normal eyes. ${ }^{12}$ Therefore, we know that alterations in vessels and in ocular circulations occur in eyes with RP. Unfortunately, these changes are clinically difficult to follow because measurement of blood flow velocity and vessel tortuosity require specific devices and/or analyses that are not easily performed in the clinical setting.
Correspondence: Akio Oishi

Department of Ophthalmology and Visual Sciences, Kyoto University Graduate

School of Medicine

54 Kawahara, Shogoin

Sakyo, Kyoto 606-8507, Japan

Tel +81757513253

Fax+8I 757520933

Email aquio@kuhp.kyoto-u.ac.jp 
Over the past decade, quantification of retinal vascular caliber, using fundus photographs, has been widely used in epidemiological studies. ${ }^{13-18}$ The Interactive Vessel Analysis (IVAN; University of Wisconsin-Madison, Madison, WI, USA) program is a well-established method to semi-automatically quantify vessel caliber. ${ }^{19,20}$ Using this program, retinal vessel analyses have been performed for patients with various ocular diseases, including diabetic retinopathy ${ }^{21}$ branch retinal vein occlusion, ${ }^{22}$ age-related macular degeneration, ${ }^{23}$ and normaltension glaucoma. ${ }^{24}$ Evidence from these reports suggests that disease pathogenesis may be linked to vascular alterations.

Recently, Ma et $\mathrm{al}^{25}$ investigated the correlation between vessel attenuation and visual function in patients with RP. However, as the authors noted, the study was cross-sectional and did not provide information on how retinal vascular caliber changes over time. Additionally, the study had other limitations, including the use of old fundus camera models with optics that may have affected vessel caliber measurement, ${ }^{26}$ the exclusion of data from $65 \%$ of subjects, a lack of control data, and the inclusion of data from both eyes of each patient.

In this study, we quantified changes in retinal vessel caliber over time in eyes with RP. The study was conducted over a 3-year interval to determine whether visual function changes were correlated with retinal vessel attenuation.

\section{Materials and methods Study population}

In this study, we enrolled 212 patients with RP who visited the Department of Ophthalmology at Kyoto University Hospital between July 2007 and June 2009. As of July 2012, 126 of these patients had been followed-up for 3 or more years. Of these 126 patients, we included baseline and 3-year interval data of those patients who underwent comprehensive ophthalmic examinations, including best-corrected visual acuity (VA) measurement, fundus photography, static visual field test (Humphrey field analyzer, standard 10-2 program), kinetic visual field test (Goldmann perimeter), and spectral domain optical coherence tomography (SD-OCT). Conventional electroretinography (ERG), recorded according to the 2008 recommendations of the International Society for Clinical Electrophysiology of Vision, ${ }^{27}$ was performed at least once for each patient. For dark-adapted ERGs (dim white stimulus flash of $0.01 \mathrm{~cd} \cdot \mathrm{s} / \mathrm{m}^{2}$, minimum interval of 2 seconds between flashes), those with undetectable responses were categorized as "non-recordable". Electroretinogram parameters that fell below the normative $95 \%$ confidence intervals were categorized as "subnormal".
RP was diagnosed based on the presence of the following: night blindness, fundus characteristics consistent with $\mathrm{RP}$, ring-shaped or concentric scotomas corresponding to the fundus abnormalities, and non-recordable/subnormal ERGs. Thirty-five of 126 patients (27.8\%) failed to undergo periodic examinations, and fundus photographs of adequate quality could not be obtained in six patients (4.7\%). Thus, the final sample consisted of 85 RP patients (67.5\%). Data from the left eye of each subject were used in analyses, unless there was significant media opacity. In these cases, data from the right eye were used when possible. To compare central retinal artery equivalent (CRAE) and central retinal vein equivalent (CRVE) results from RP patients, age- and sex-matched normal subjects were selected by using archived fundus photographs from our department's library. Since all the participants were Japanese, control subjects were selected from a Japanese population.

\section{Retinal vessel caliber measurement}

A disc-centered photograph was obtained with a digital camera (TRC NW6SF; Topcon Corp., Tokyo, Japan) at baseline and each follow-up visit. Retinal vascular caliber was measured by three trained graders using a computerbased program, IVAN, and following established, standardized protocols. ${ }^{19,20}$ All graders were masked to ocular status (RP or control) and ocular examination results of the subjects. All arterioles and venules within one-half to one disc diameter of the optic disc margin were measured (Figure 1). The diameters of the six largest arteries and veins were then used to calculate CRAE and CRVE, respectively. Before the measurements, 100 standard images were randomly selected and analyzed to assess the intra- and inter-grader reliability and the intraclass and interclass reproducibility. The intraclass and interclass correlation coefficients (ICC [1.1]) were 0.907 for CRAE and 0.996 for CRVE. The inter-grader reliability (ICC [3.1]) was 0.854 for CRAE and 0.987 for CRVE. Since the measurement of the six largest vessels was not possible in some cases, we also evaluated the clinical significance of the mean of the two largest arterioles/venules.

\section{Optical coherence tomography (OCT) acquisition and analysis}

At baseline and all follow-up visits, patients underwent a dilated OCT examination with SD-OCT (Spectralis ${ }^{\circledR}$ HRA + OCT; Heidelberg Engineering, Heidelberg, Germany), performed by an experienced technician. All images were obtained using the built-in eye-tracking system, and 100 scans were automatically averaged to reduce the 

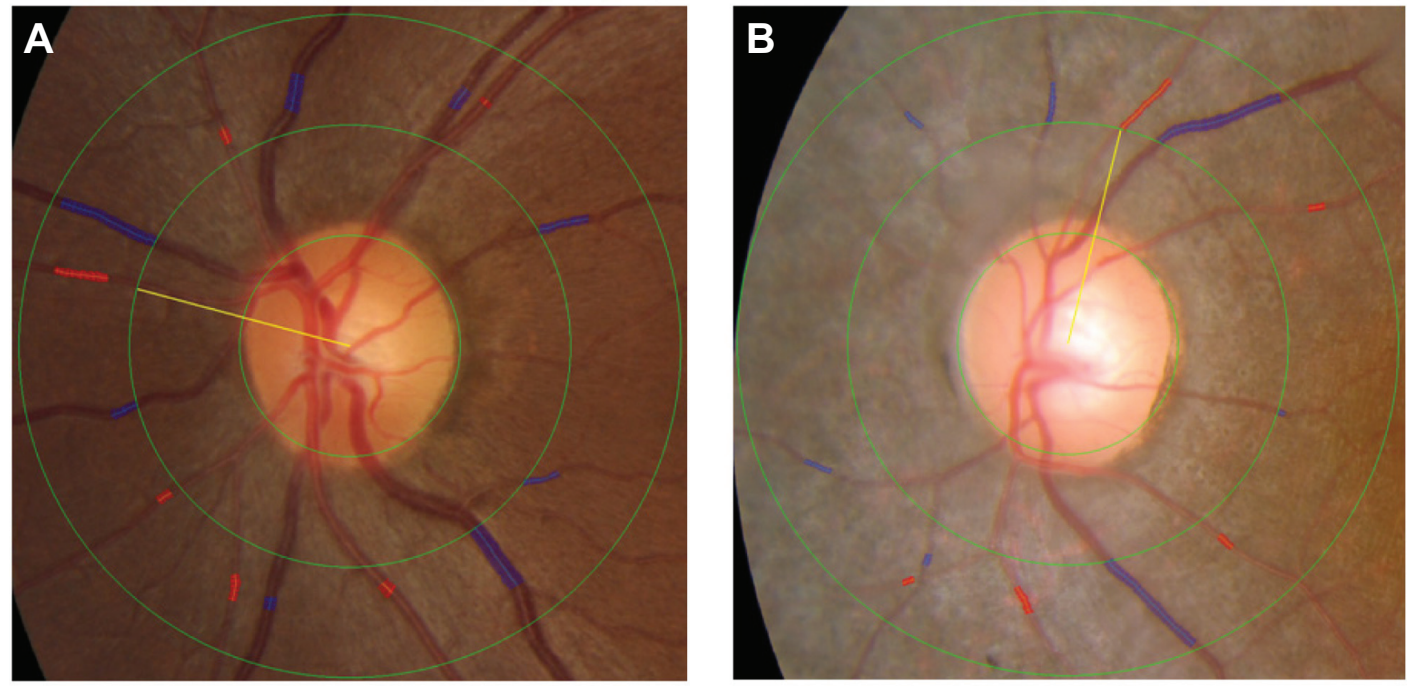

Figure I Identification and measurement of the retinal arteriole and venule caliber using the IVAN semi-automated computer imaging program.

Notes: The blue and red shading indicates the selected venule and arteriole area, respectively, used to determine vessel caliber. (A) Color photograph of the left eye of a 32-year-old woman in the control group. More than six venules and arterioles could easily be measured in all normal patients. (B) Color photograph of the left eye of a 22-year-old woman with retinitis pigmentosa. Seven venules and only five arterioles could be measured.

Abbreviation: IVAN, interactive vessel analysis.

signal-to-noise ratio. The central macular thickness (CMT) was defined as the vertical distance between the vitreoretinal interface and the outer border of the retinal pigment epithelium, using the horizontal and vertical line scans through the foveal center. All measurements were performed manually using the SD-OCT built-in calibers. Macular thickness was determined by averaging macular thickness measurements from the vertical and horizontal OCT scans.

\section{Humphrey field analyzer 10-2 program acquisition and analysis}

A static visual field test (Swedish Interactive Threshold Algorithm standard 10-2 program) was performed with the Humphrey field analyzer (Carl Zeiss Meditec AG, Jena, Germany). Mean deviation (MD), which is a weighted average of visual threshold at each measurement position, was used for the analysis. When the fixation loss exceeded $20 \%$ or the false-positive or false-negative error rates were over $25 \%$, data were excluded from the study.

\section{Visual field examination}

Visual field examination (V4e, white test light, Goldmann perimeter; Haag Streit, Bern, Switzerland) results were digitally scanned and then analyzed using Image J software (US National Institutes of Health, Bethesda, MD, USA; downloaded from http://imagejnihgov/ij/). First, the magnification scale was calibrated using the radius of the central $30^{\circ}$ circle on the Goldmann perimeter report. With this calibration, it was determined that 140,500 pixels was equivalent to a scanned area of $40.69 \mathrm{~cm}^{2}$ on the visual field recording paper. The remaining visual field area, defined by the V4e isopter, was measured using the "Measurement" function of the Image $\mathrm{J}$ software.

\section{Ethics approval}

This study was performed in accordance with the tenets of the Declaration of Helsinki, and was approved by the Institutional Review Board/Ethics Committee of the Kyoto Graduate School of Medicine (Kyoto, Japan). Because only medical records were reviewed, this retrospective study received exempt status and it was not mandatory to obtain informed consent from RP or healthy control subjects.

\section{Data and statistical analyses}

It is well-known that RP has bilateral symmetry, ${ }^{28}$ therefore, measurements in one eye should be representative of the fellow eye. Here, data from the left eye of each patient were used in analyses whenever possible. If media opacities in the left eye prevented adequate imaging from being obtained, data from the right eye were used. Data are presented as either mean \pm standard deviation or as absolute numbers (percentages).

For statistical analyses, VA was measured using the Landolt $\mathrm{C}$ test and converted to the logarithm of the minimum angle of resolution (logMAR) equivalent. The unpaired $t$-test 
was used to compare continuous variables, and the chi-square test was used to compare categorical variables between groups, as appropriate. Correlations among parameters were tested for statistical significance using Pearson's correlation test. To adjust for confounding factors, a stepwise linear regression model was used, with either CRAE or CRVE as the dependent variable and with age, VA, visual field area, $\mathrm{MD}$, and CMT as the independent variables. Analyses were performed using SPSS software (version 19.0; IBM Japan, Tokyo, Japan). Statistical significance was defined as a $P$-value less than 0.05 .

\section{Results}

\section{Descriptive data}

Of the 85 RP eyes included, the six largest arterioles and venules were gradable in only 45 eyes $(52.9 \%)$. In the remaining $40 \mathrm{RP}$ patients $(47.1 \%)$, we could not calibrate the six arterioles, but calibration of the six venules was possible in all cases (Figure 1).

\section{Correlations of the baseline parameters}

Table 1 summarizes the baseline characteristics of the RP and control groups. Two-thirds of RP patients were female, and their mean age at baseline was 45.0 \pm 16.1 years. Mean CRAE was significantly smaller in RP patients $(94.9 \pm 13.5 \mu \mathrm{m})$ than in the control group $(138.1 \pm 14.7 \mu \mathrm{m}, P<0.0001)$, as was CRVE (155.6 $\pm 20.0 \mu \mathrm{m}$ versus $215.0 \pm 20.4 \mu \mathrm{m}$, $P<0.0001)$. There were no significant differences in subject history, including diabetes, hypertension, hyperlipidemia, heart disease, cardiovascular disease, or smoking habits. The clinical baseline characteristics of the patients with RP are shown in Table 2. The mean age at disease onset was $27.4 \pm 16.9$ years, and the mean disease duration, defined as the time from onset of symptoms to the day of examination, was $17.6 \pm 14.3$ years.

\section{Correlations of the follow-up parameters}

Disease progression was noted on each examination every 3 years, as was a decline in visual function (Table 3 ). VA, MD, visual field area, and retinal vessel caliber also showed statistically significant worsening at each 3-year interval. The relationships between various ocular characteristics and CRAE/CRVE are shown in Table 4. At the 3-year follow-up, visual field area was significantly associated with both CRAE ( $r=0.584, P=0.001)$ and CRVE ( $r=0.500, P=0.008$ ), and the MD was significantly associated with CRAE $(r=0.298, P=0.047)$. Multivariate linear regression analysis showed that each $0.12 \mu \mathrm{m}$ decrease in CRAE corresponded to $1 \mathrm{~cm}^{2}$ of visual field constriction in RP patients. Other factors were excluded with stepwise analysis. We performed the same analyses on baseline vessel caliber and clinical characteristics, which showed very similar results.

\section{Correlations of vessel baseline and functional changes parameters}

We next investigated associations between changes in VA, CMT, visual field area, MD, and changes in CRAE and CRVE. However, there were no significant associations, partly because of wide data dispersion. We also investigated the association between baseline retinal vessel caliber and visual function changes. Baseline vessel caliber (CRAE and CRVE) was inversely associated with VA decline (Figure 2), indicating that smaller retinal vessels predicted larger declines in VA over 3 years. There was no significant correlation with the other measurements.

Table I Baseline demographics of participants with retinitis pigmentosa and healthy controls

\begin{tabular}{llll}
\hline Analyzed parameters & RP & Age-sex matched control & P-value \\
\hline Number & 45 & 45 & $15 / 30$ \\
Sex (male/female) & $15 / 30$ & $45.1 \pm 20.7$ & 1 \\
Age (years) & $45.0 \pm 16.1$ & $138.1 \pm 14.7$ & 0.973 \\
CRAE $(\mu \mathrm{m})$ & $94.9 \pm 13.5$ & $215.0 \pm 20.4$ & $<0.000 \mathrm{I}$ \\
CRVE $(\mu \mathrm{m})$ & $155.5 \pm 20.0$ & $0.6 \pm 0.1$ & $<0.000 \mathrm{I}$ \\
AVR & $0.6 \pm 0.1$ & $3(6.7 \%)$ & 0.012 \\
Diabetes, no. $(\%)$ & 0 & $3(6.7 \%)$ & $\mathrm{NA}$ \\
Hypertension, no. $(\%)$ & $7(15.6 \%)$ & $4(8.9 \%)$ & 0.314 \\
Hyperlipidemia & $2(4.4 \%)$ & $4(8.9 \%)$ & 0.673 \\
Heart disease & $2(4.4 \%)$ & $1(2.2 \%)$ & 0.673 \\
Cardiovascular disease & $\mathrm{I}(2.2 \%)$ & $9(20.0 \%)$ & $\mathrm{I}$ \\
Past or current cigarette smoking, no. $(\%)$ & $12(26.7 \%)$ & 0.618 \\
\hline
\end{tabular}

Notes: Data are means \pm standard deviations; unpaired $t$-test for continuous variables or chi-square test for categorical variables, as appropriate.

Abbreviations: AVR, arteriovenous ratio; CRAE, central retinal artery equivalent-6; CRVE, central retinal vein equivalent-6; NA, not applicable; no., number; RP, retinitis pigmentosa. 
Table 2 Clinical baseline characteristics of study subjects $(n=45)$

\begin{tabular}{llll}
\hline Characteristics & & $\mathbf{n}$ & $\mathbf{( \% )}$ \\
\hline Mode of inheritance & Autosomal recessive & 12 & $26.7 \%$ \\
& Autosomal dominant & 6 & $13.3 \%$ \\
& Sporadic & 27 & $60.0 \%$ \\
ERG & Subnormal & 2 & $4.4 \%$ \\
& Non-recordable & 43 & $95.6 \%$ \\
& & Mean & SD \\
Estimated age of onset & (Years old) & 27.4 & 16.9 \\
Estimated duration & (Years) & 17.6 & 14.3 \\
\hline
\end{tabular}

Abbreviations: ERG, electroretinogram; SD, standard deviation.

Finally, to include the patients in whom six arterioles could not be calibrated, CRAE and CRVE were recalculated, in all $85 \mathrm{RP}$ patients, as the average diameter of the two largest arterioles and veins, respectively. The associations between the mean of largest two arterioles/veins and each parameter are summarized in Table 5. Interestingly, these values were also associated with visual field area and, in addition, VA and MD. These results suggest that even the caliber of the largest two vessels, which were always arcade vessels, can predict visual changes in RP patients.

\section{Discussion}

In this study, we confirmed that retinal vessels significantly narrow over a 3-year interval in eyes with RP. We also show that retinal vascular caliber attenuation is correlated with some visual functions. In addition, our results suggest that baseline vessel caliber may be predictive of VA decline in the following 3 years.

Consistent with a previous report, ${ }^{25}$ both CRAE and CRVE were significantly correlated with visual field area. While another study showed the association between macular thickness and retinal vessel caliber in healthy individuals, ${ }^{29}$ this association was not confirmed in our cohort. These results

Table 3 Mean change in visual function over 3-year intervals in study population with RP

\begin{tabular}{llll}
\hline Variable & Baseline & Follow-up & $P$-value \\
\hline CRAE & $94.9 \pm 2.0$ & $90.7 \pm 1.9$ & 0.001 \\
CRVE & $155.6 \pm 3.0$ & $152.3 \pm 2.8$ & 0.014 \\
AVR & $0.6 \pm 0.009$ & $0.6 \pm 0.010$ & 0.086 \\
Visual acuity (logMAR) & $0.10 \pm 0.04$ & $0.17 \pm 0.05$ & 0.008 \\
Mean deviation & $-15.6 \pm 1.3$ & $-16.7 \pm 1.4$ & $<0.0001$ \\
CMT & $226.0 \pm 11.5$ & $209.4 \pm 12.2$ & 0.013 \\
Visual field area (V4e) & $103.0 \pm 14.9$ & $77.9 \pm 13.6$ & $<0.0001$
\end{tabular}

Notes: Data are means \pm standard deviations; paired $t$-test for continuous variables, as appropriate.

Abbreviations: AVR, arteriovenous ratio; CMT, central macular thickness; CRAE, central retinal artery equivalent; CRVE, central retinal vein equivalent; $\log M A R$, logarithm of the minimum angle of resolution; RP, retinitis pigmentosa; visual field area (V4e), Goldmann visual field test area for V4e.
Table 4 Relationship of various characteristics to central retinal arteriolar equivalent and central retinal venular equivalent in subjects with retinitis pigmentosa

\begin{tabular}{llllll}
\hline Characteristic & \multicolumn{2}{l}{$\begin{array}{l}\text { Central retinal } \\
\text { arteriolar equivalent }\end{array}$} & & \multicolumn{2}{l}{$\begin{array}{l}\text { Central retinal } \\
\text { venular equivalent }\end{array}$} \\
\cline { 2 - 3 } \cline { 5 - 6 } & $\boldsymbol{P}$-value & $\boldsymbol{r}$ & & $\boldsymbol{P}$-value & $\boldsymbol{r}$ \\
\hline Visual acuity (logMAR) & 0.092 & -0.254 & & 0.083 & -0.262 \\
Mean deviation & 0.047 & 0.298 & & 0.092 & 0.254 \\
Visual field area (V4e) & 0.001 & 0.584 & & 0.008 & 0.500 \\
CMT & 0.536 & 0.095 & & 0.314 & 0.154 \\
\hline
\end{tabular}

Abbreviations: CMT, central macular thickness; logMAR, logarithm of the minimum angle of resolution; $r$, Pearson's correlation coefficient; visual field area (V4e), Goldmann visual field test area for $\mathrm{V} 4 \mathrm{e}$.

provide further information on retinal circulation and retinal morphology or function in eyes with RP.

The mechanisms underlying vascular attenuation in the diseased retina remain unclear. One hypothesis is that after photoreceptor death, the oxygen requirement of the outer retina decreases and excessive oxygen reaches the inner retina causing a hyperoxic state, which in turn results in vasoconstriction. ${ }^{30-32}$ Another hypothesis suggests that primary photoreceptor death sequentially causes thinning of the outer retina. This could result in thickening of the extracellular matrix between the migrated retinal pigment epithelium cells and the retinal vessels and narrowing of the retinal vasculature. ${ }^{1,8}$ Alternatively, Stone et a ${ }^{33}$ suggested that after photoreceptor death, synaptic input is lost and the decline in trophic factors causes a decreased inner retinal metabolism. This may induce vascular remodeling and subsequent decreases in blood supply to the residual retinal tissue. ${ }^{33}$ In this study, whereas retinal function was associated with vessel caliber at given time points, neither changes in retinal function nor macular thickness were associated with vascular alteration in the 3 -year period. Therefore, it is likely that these processes do not occur in parallel. Although the result does not provide conclusive evidence, the first of the above hypotheses is less likely to be the primary one. Considering that oxygen levels are expected to change promptly after photoreceptor death, the subsequent change in vessel caliber should be more closely associated with retinal changes.

VA tended to decline more in patients with narrower baseline vessels. Although we believe that retinal vessel attenuation fundamentally results from photoreceptor degeneration, there may be a mechanism that attenuates vessels. This may induce further photoreceptor cell death by loss of trophic factors, as already suggested by Stone et al. ${ }^{33}$ Interestingly, modulation of retinal vessels seems to protect photoreceptors in a mouse model of RP. ${ }^{34}$ Alternatively, attenuated vessels 

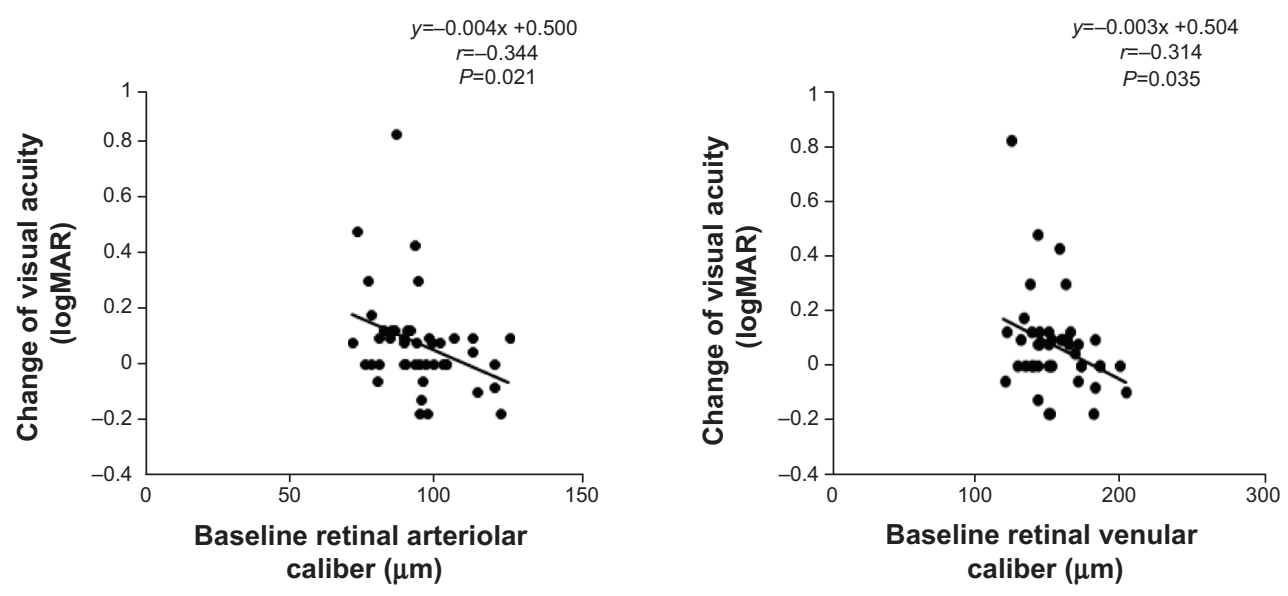

Figure 2 Correlation between baseline retinal vessel caliber and the changes in visual acuity in patients with retinitis pigmentosa.

Notes: A positive number of visual acuity changes represents deterioration. Narrower central retinal arteriolar equivalent values at baseline were associated with bigger changes in visual acuity over each 3-year interval.

Abbreviation: logMAR, logarithm of the minimum angle of resolution.

indicate a more hyperoxic state and higher levels of oxidative stress, which is known to be toxic to photoreceptors. ${ }^{35}$ Further investigations are needed to clarify these issues.

Measurement of six arterioles is sometimes difficult in eyes with RP, especially in advanced cases. These eyes would likely be excluded from clinical trials, but are often seen in clinical practice. Therefore, we investigated whether or not manually calculating CRAE and CRVE with just two arteriole or venule vessel measurements is useful in these patients. The mean diameters of the two largest arteries and venules were well correlated with visual function and retinal morphology. These results suggest these manual CRAE and CRVE values may be a useful alternative in clinical practice, even in more advanced disease states.

To the best of our knowledge, this is the first report of correlations between changes in visual function over time and retinal vessel caliber. However, this study has several limitations. First, systemic blood pressure and blood glucose were not measured at the time of retinal photography and imaging. These systemic parameters can alter retinal vascular

Table 5 Relationship of mean of two largest arterioles or venules to variable parameters

\begin{tabular}{llllll}
\hline Characteristic & \multicolumn{2}{l}{$\begin{array}{l}\text { Mean of two } \\
\text { largest arterioles }\end{array}$} & & \multicolumn{2}{l}{$\begin{array}{l}\text { Mean of two } \\
\text { largest venules }\end{array}$} \\
\cline { 2 - 3 } & $\boldsymbol{P}$-value & $\boldsymbol{r}$ & & P-value & $\boldsymbol{r}$ \\
\hline Visual acuity (logMAR) & 0.010 & -0.277 & & 0.002 & -0.325 \\
Mean deviation & 0.003 & 0.322 & & 0.0001 & 0.404 \\
Visual field area (V4e) & 0.030 & 0.419 & & 0.008 & 0.489 \\
CMT & 0.985 & -0.002 & & 0.889 & -0.015 \\
\hline
\end{tabular}

Abbreviations: CMT, central macular thickness; logMAR, logarithm of the minimum angle of resolution; $r$, Pearson's correlation coefficient; visual field area (V4e), Goldmann visual field test area for $\mathrm{V} 4 \mathrm{e}$. caliber, ${ }^{36}$ which could have affected the results. Second, this study was performed using retrospective data, and we cannot determine whether retinal vessel attenuation precedes or follows retinal degeneration. In summary, quantitative assessment of retinal vessel caliber may be useful in assessing the RP stage and predicting VA changes.

\section{Acknowledgments}

The authors thank Atsushi Otani, MD, PhD, for helpful advice on this manuscript and are grateful to Professor Nicola Ferrier (Department of Mechanical Engineering, University of Wisconsin-Madison) for generously providing the IVAN analysis program.

\section{Disclosure}

The authors report no conflicts of interest in this work.

\section{References}

1. Li ZY, Possin DE, Milam AH. Histopathology of bone spicule pigmentation in retinitis pigmentosa. Ophthalmology. 1995;102(5):805-816.

2. Merin S. Retinitis pigmentosa. In: Merin S, editor. Inherited Eye Diseases: diagnosis and clinical management. 1st ed. New York: Marcel Dekker Inc.; 1991:219-279.

3. Green WR. Primary pigmentary Degeneration of the Retina In Spencer WH, editor. Ophthalmic Pathology: An Atlas and Textbook. 4th edition, vol 2, Philadelphia: W.B. Saunders Company; 1996:1263-1268.

4. Akyol N, Kükner S, Celiker U, Koyu H, Lüleci C. Decreased retinal blood flow in retinitis pigmentosa. Can J Ophthalmol. 1995;30(1):28-32.

5. Grunwald JE, Maguire AM, Dupont J. Retinal hemodynamics in retinitis pigmentosa. Am J Ophthalmol. 1996;122(4):502-508.

6. Beutelspacher SC, Serbecic N, Barash H, et al. Retinal blood flow velocity measured by retinal function imaging in retinitis pigmentosa. Graefes Arch Clin Exp Ophthalmol. 2011;249(12):1855-1858.

7. Falsini B, Anselmi GM, Marangoni D, et al. Subfoveal choroidal blood flow and central retinal function in retinitis pigmentosa. Invest Ophthalmol Vis Sci. 2011;52(2):1064-1069. 
8. Cellini M, Strobbe E, Gizzi C, Campos EC. ET-1 plasma levels and ocular blood flow in retinitis pigmentosa. Can J Physiol Pharmacol. 2010;88(6):630-635.

9. Muir ER, De La Garza B, Duong TQ. Blood flow and anatomical MRI in a mouse model of retinitis pigmentosa. Magn Reson Med. 2013;69(1): 221-228.

10. Li G, De La Garza B, Shih YY, Muir ER, Duong TQ. Layer-specific bloodflow MRI of retinitis pigmentosa in RCS rats. Exp Eye Res. 2012;101: 90-96.

11. Schmidt KG, Pillunat LE, Kohler K, Flammer J. Ocular pulse amplitude is reduced in patients with advanced retinitis pigmentosa. $\mathrm{Br} J$ Ophthalmol. 2001;85(6):678-682.

12. Dougherty G, Johnson MJ, Wiers MD. Measurement of retinal vascular tortuosity and its application to retinal pathologies. Med Biol Eng Comput. 2010;48(1):87-95.

13. Wong TY, Klein R, Sharrett AR, et al. Retinal arteriolar narrowing and risk of coronary heart disease in men and women. The Atherosclerosis Risk in Communities Study. JAMA. 2002;287(9):1153-1159.

14. Wong TY, Shankar A, Klein R, Klein BE, Hubbard LD. Retinal arteriolar narrowing, hypertension, and subsequent risk of diabetes mellitus. Arch Intern Med. 2005;165(9):1060-1065.

15. Wong TY, Kamineni A, Klein R, et al. Quantitative retinal venular caliber and risk of cardiovascular disease in older persons: the cardiovascular health study. Arch Intern Med. 2006;166(21):2388-2394.

16. Lindley RI, Wang JJ, Wong MC, et al; Multi-Centre Retina and Stroke Study (MCRS) Collaborative Group. Retinal microvasculature in acute lacunar stroke: a cross-sectional study. Lancet Neurol. 2009;8(7): 628-634.

17. McGeechan K, Liew G, Macaskill P, et al. Prediction of incident stroke events based on retinal vessel caliber: a systematic review and individual-participant meta-analysis. Am J Epidemiol. 2009;170(11): 1323-1332.

18. McGeechan K, Liew G, Macaskill P, et al. Meta-analysis: retinal vessel caliber and risk for coronary heart disease. Ann Intern Med. 2009; 151(6):404-413.

19. Knudtson MD, Lee KE, Hubbard LD, Wong TY, Klein R, Klein BE. Revised formulas for summarizing retinal vessel diameters. Curr Eye Res. 2003;27(3):143-149.

20. Hubbard LD, Brothers RJ, King WN, et al. Methods for evaluation of retinal microvascular abnormalities associated with hypertension/ sclerosis in the Atherosclerosis Risk in Communities Study. Ophthalmology. 1999;106(12):2269-2280.

21. Klein R, Klein BE, Moss SE, Wong TY. Retinal vessel caliber and microvascular and macrovascular disease in type 2 diabetes: XXI: the Wisconsin Epidemiologic Study of Diabetic Retinopathy. Ophthalmology. 2007;114(10):1884-1892.

22. Youm DJ, Ha MM, Chang Y, Song SJ. Retinal vessel caliber and risk factors for branch retinal vein occlusion. Curr Eye Res. 2012;37(4) 334-338.
23. Jeganathan VS, Kawasaki R, Wang JJ, et al. Retinal vascular caliber and age-related macular degeneration: the Singapore Malay Eye Study. Am J Ophthalmol. 2008;146(6):954-959.e1.

24. Chang M, Yoo C, Kim SW, Kim YY. Retinal vessel diameter, retinal nerve fiber layer thickness, and intraocular pressure in Korean patients with normal-tension glaucoma. Am J Ophthalmol. 2011;151(1): 100-105.e1.

25. Ma Y, Kawasaki R, Dobson LP, et al. Quantitative analysis of retinal vessel attenuation in eyes with retinitis pigmentosa. Invest Ophthalmol Vis Sci. 2012;53(7):4306-4314.

26. Pauli TW, Gangaputra S, Hubbard LD, et al. Effect of image compression and resolution on retinal vascular caliber. Invest Ophthalmol Vis Sci. 2012;53(9):5117-5123.

27. Marmor MF, Fulton AB, Holder GE, Miyake Y, Brigell M, Bach M; International Society for Clinical Electrophysiology of Vision. ISCEV Standard for full-field clinical electroretinography (2008 update). Doc Ophthalmol. 2009;118(1):69-77.

28. Massof RW, Finkelstein D, Starr SJ, Kenyon KR, Fleischman JA, Maumenee IH. Bilateral symmetry of vision disorders in typical retinitis pigmentosa. Br J Ophthalmol. 1979;63(2):90-96.

29. Samarawickrama C, Huynh SC, Wang JJ, et al. Relationship between retinal structures and retinal vessel caliber in normal adolescents. Invest Ophthalmol Vis Sci. 2009;50(12):5619-5624.

30. Padnick-Silver L, Kang Derwent JJ, Giuliano E, Narfstrom K, Linsenmeier RA. Retinal oxygenation and oxygen metabolism in Abyssinian cats with a hereditary retinal degeneration. Invest Ophthalmol Vis Sci. 2006;47(8):3683-3689.

31. Yu DY, Cringle SJ. Retinal degeneration and local oxygen metabolism. Exp Eye Res. 2005;80(6):745-751.

32. Penn JS, Li S, Naash MI. Ambient hypoxia reverses retinal vascular attenuation in a transgenic mouse model of autosomal dominant retinitis pigmentosa. Invest Ophthalmol Vis Sci. 2000;41(12):4007-4013.

33. Stone JL, Barlow WE, Humayun MS, de Juan E Jr, Milam AH. Morphometric analysis of macular photoreceptors and ganglion cells in retinas with retinitis pigmentosa. Arch Ophthalmol. 1992;110(11): 1634-1639.

34. Otani A, Dorrell MI, Kinder K, et al. Rescue of retinal degeneration by intravitreally injected adult bone marrow-derived lineage-negative hematopoietic stem cells. J Clin Invest. 2004;114(6):765-774.

35. Komeima K, Rogers BS, Lu L, Campochiaro PA. Antioxidants reduce cone cell death in a model of retinitis pigmentosa. Proc Natl Acad Sci US A. 2006;103(30):11300-11305.

36. Sun C, Wang JJ, Mackey DA, Wong TY. Retinal vascular caliber: systemic, environmental, and genetic associations. Surv Ophthalmol. 2009; 54(1):74-95.
Clinical Ophthalmology

\section{Publish your work in this journal}

Clinical Ophthalmology is an international, peer-reviewed journal covering all subspecialties within ophthalmology. Key topics include: Optometry; Visual science; Pharmacology and drug therapy in eye diseases; Basic Sciences; Primary and Secondary eye care; Patient Safety and Quality of Care Improvements. This journal is indexed on Submit your manuscript here: http://www.dovepress.com/clinical-ophthalmology-journal

\section{Dovepress}

PubMed Central and CAS, and is the official journal of The Society of Clinical Ophthalmology (SCO). The manuscript management system is completely online and includes a very quick and fair peer-review system, which is all easy to use. Visit http://www.dovepress.com/ testimonials.php to read real quotes from published authors. 